\title{
GARANTIA DA QUALIDADE DO CAFÉ SOLÚVEL COM O USO DO GRÁFICO DE CONTROLE DE SOMAS ACUMULADAS
}

\section{QUALITY ASSURANCE IN THE PRODUCTION OF INSTANT COFFEE USING THE CUSUM CONTROL CHART}

\author{
Wesley Vieira da Silva, Dr. \\ Professor do Programa da Pós-Graduação Stricto Sensu em Administração da PUCPR \\ Rua Imaculada Conceição, 1155, Bloco Acadêmico - Mestrado em Administração \\ Estratégica, Campus Curitiba, Prado Velho, Curitiba-PR. CEP: 80215-901. E-mail: \\ Telefone: (41) 3271-1476 e Fax: (41) 3271-1638 / wesley.vieira@ pucpr.br
}

\section{Carlos Augusto Candêo Fontanini, M. Sc.}

Professor e Coordenador do Curso de Administração da PUCPR

Rua Imaculada Conceição, 1155, Bloco Acadêmico - Coordenação do Curso de Graduação em Administração, Campus Curitiba, Prado Velho, Curitiba-PR. CEP: 80215-901. E-mail:

Telefone: (41) 3271-1476 e Fax: (41) 3271-1638 / c.fontanini@pucpr.br

\section{Jansen Maia Del Corso, Dr.}

Professor do Programa da Pós-Graduação Stricto Sensu em Administração da PUCPR

Rua Imaculada Conceição, 1155, Bloco Acadêmico - Mestrado em Administração

Estratégica, Campus Curitiba, Prado Velho, Curitiba-PR. CEP: 80215-901. E-mail::

Telefone: (41) 3271-1476 e Fax: (41) 3271-1638 / del.corso@pucpr.br

\section{RESUMO}

Este trabalho tem por objetivo analisar o nível de estabilidade e garantia da qualidade do processo de fabricação de café solúvel em uma indústria no Estado do Paraná. A amostra coletada corresponde a 4.598 observações do peso de latas de $50 \mathrm{~g}$ e $100 \mathrm{~g}$ de café instantâneo em pó, sendo realizada de forma computadorizada no período de 8 de outubro a 5 de dezembro de 2004. Os resultados auferidos, com base nos resíduos provenientes dos modelos autoregressivos de terceira ordem (ARIMA), foram avaliados com base na carta de controle de somas acumuladas (CUSUM) a qual evidenciou um processo produtivo instável 
estatisticamente, o que corrobora com uma fragilidade do processo no que tange à garantia de produtos não conformes, principalmente quanto ao peso dos vasilhames de $50 \mathrm{~g}$. Os resultados apresentados propõem maior atenção por parte dos funcionários da indústria de fabricação de café, dispensando maior disciplina e controle nas atividades realizadas no dia-a-dia, além de permitir dizimar importantes erros cometidos na rotina de trabalho e que podem ser solucionados com boa orientação e mudança de comportamento no que tange à regulagem e manutenção dos equipamentos.

Palavras Chave: ARIMA, Somas Acumuladas e Café.

\begin{abstract}
This paper aims to evaluate stability and quality guarantee of the productive process regarding soluble coffee plant situated in the state of Paraná. The collected sample contents 4.598 observations of weight of 50 and 100 grams cans. The process of collection was made automatically during period of October $8^{\text {th }}$ and December $5^{\text {th }}$ 2004. ARIMA model's residues were evaluated by the cumulated sum control charts (CUSUM) with "V" mask form, which showed that productive process is statistically unstable. This is evinces fragility of the process regarding the guarantee for the products that do not comply with requirements, mainly the weight of the 50 grams' containers. The results suggest that employees should have more accurate attention to the discipline and control of everyday activities, besides banning mistakes made by them on the routine work that can be solved with the right orientation and changing equipment adjustment procedures.

Key Words: ARIMA, Cumulated Cum Control Charts (CUSUM), Soluble Coffee Productive Process.
\end{abstract}

\title{
1- INTRODUÇÃO
}

Ao ser analisada uma determinada peça numa linha de produção, percebe-se a existência de algum grau de variabilidade de um produto para outro, normalmente apresentando diferenças em suas formas ou dimensões. À medida que são melhoradas as performances de máquinas e equipamentos, surgem concomitantemente novos requisitos de projetos de produtos, com exigências de exatidões cada vez maiores e com mais freqüência.

Diversas vezes o próprio operador de determinado sistema produtivo introduz variação adicional ao mesmo, o que acaba provocando não conformidades nos produtos elaborados $a$ 
posteriori. Deming (1986) afirma que mesmo a melhor máquina automática não pode produzir todas as unidades exatamente iguais. Vale salientar que o operador, ao reagir diante de episódios localizados, deixa de focalizar o sistema produtivo como um todo, onde está a maior parte das causas de variações e de possíveis erros provenientes da produção.

Assim, é preciso que o operador saiba identificar com clareza as variabilidades que estejam ocorrendo, buscando com isso suas causas e tomando as devidas ações corretivas, dado que à medida que a variabilidade é reduzida no processo, a qualidade dos produtos é melhorada. As ações preventivas no processo fazem ainda com que os itens fabricados não sejam expedidos e remetidos aos potenciais clientes, o que acaba promovendo ganhos ou perdas de oportunidades para a empresa.

Nesses termos, o Controle Estatístico de Processos (CEP) pode ser usado para controlar as variabilidades de processos produtivos. Adotam-se os gráficos ou cartas de controle que buscam monitorar as características dos produtos com relação aos limites de tolerância ou de especificação. Esses gráficos, também conhecidos na literatura como cartas de controle, buscam encontrar variabilidades no processo, aumentando por conseqüência, a produtividade e prevenindo, possíveis ajustes desnecessários nos equipamentos monitorados.

A indústria analisada é considerada um veículo de competitividade nesse setor de atividade econômica e está procurando avaliar o comportamento do seu processo produtivo ${ }^{\mathrm{i}}$, mais precisamente o processo de produção de café solúvel. Este estudo visa detectar possíveis anomalias no processo, recolocando, conseqüentemente, o sistema em condições estáveis, de modo a garantir a qualidade do produto.

A justificativa pela escolha do processo produtivo de café a ser analisado se deve ao fato de esse produto possui um forte apelo emocional, além de saber que o parque cafeicultor brasileiro, em função de sua variedade e complexidade, apresenta plenas condições de atendimento aos diferentes tipos de necessidades dos consumidores nacionais e internacionais.

Por outro lado, a escolha da carta de controle estatístico de processos de Somas Acumuladas (CUSUM) se deve ao fato de que as cartas convencionais de Shewhart não serem capazes de captar pequenas variações no processo produtivo, o que não ocorre com a carta de controle CUSUM, já que ela possui a vantagem de ter memória curta, uma vez que leva em conta as informações do período imediatamente anterior.

Este trabalho está estruturado em cinco seções. A segunda seção traz uma breve revisão de literatura sobre a carta de controle de Somas Acumuladas (CUSUM). A terceira seção descreve brevemente os aspectos metodológicos onde se encontram incorporados a 
coleta e o tratamento dos dados, a descrição do processo produtivo e a caracterização da pesquisa. A quarta traz a análise e discussão dos resultados empíricos, e a quinta seção mostra as considerações finais e recomendações para trabalhos futuros.

\section{2- A CARTA DE CONTROLE DE SOMAS ACUMULADAS (CUSUM)}

O controle estatístico de processos consiste na coleta, análise e interpretação de dados, estabelecimento de padrões, comparação de desempenhos, verificação de desvios, tudo isso para a utilização nas atividades de melhoria e controle da qualidade de produtos e serviços. Sua ferramenta básica é a carta de controle, desenvolvida por Shewhart (1931) apud Reynolds et al. (1990), com o objetivo de controlar a variabilidade dos processos.

Shewhart desenvolveu as cartas da média $(\bar{X})$, da amplitude $(\mathrm{R})$ e do desvio padrão $(\mathrm{S})$ que, normalmente, são aplicadas a processos contínuos e semicontínuos, onde uma dimensão ou característica do produto é medida em uma amostra aleatória tirada sob um determinado período de tempo, com o objetivo de avaliar a estabilidade ou estado de controle estatístico de um processo. Segundo Hradesky (1989), a principal vantagem proporcionada pelo (CEP) é a realimentação do processo em tempo real. Essa realimentação deve ser contínua e estatisticamente fundamentada. Desse modo, em todo processo produtivo existem pontos específicos que são determinantes para que o fluxo de produção não apresente problemas.

A produção em série da peça ou a repetição do serviço traz algum grau de variação, bem como certa dispersão dos resultados ao redor de um valor nominal, chamado comumente na literatura de causa comum ou aleatória. Caso contrário, quando a mudança passa a ser não mais aleatória, mas associada a alguma causa especial que foge ao controle de quem está acompanhando o processo, isso passa a ser um problema e deve ser rapidamente corrigido.

Apesar de amplamente usadas nas indústrias, essas cartas não possuem memória; ou seja, só levam em consideração as informações obtidas no período presente. Assim, qualquer mudança que tenha ocorrido anteriormente, simplesmente é ignorada, fazendo com que as cartas de Shewhart não sejam capazes de detectar pequenas variações. Por essa razão, Page (1954) op. cit. Reynolds et al. (1990) desenvolveu a carta CUSUM, que possui uma memória curta, ou seja, leva em consideração as informações obtidas no período imediatamente anterior, além de captar mudanças pequenas, contudo persistentes, no processo avaliado.

Costa, Epprecht e Carpinetti (2004) mostram que a carta de somas acumuladas, além de sinalizar o desajuste no processo, ainda informa quando ele ocorreu. $\mathrm{O}$ fato de a carta 
CUSUM se basear em dados históricos do processo e não somente na última observação, naturalmente, não sinaliza os desajustes de imediato, independentemente de sua magnitude. Portanto, para grandes desvios da média, a carta da média $(\overline{\mathrm{X}})$ será sempre mais ágil do que a CUSUM.

A carta CUSUM considera que as observações $\mathrm{X}_{\mathrm{j}}$, com $\mathrm{j}=1,2,3, \ldots, \mathrm{n}$, sejam estatisticamente independentes e identicamente distribuídas (i.i.d.), seguindo uma distribuição normal. Logo, determina-se a soma acumulada dos desvios da média amostral $\left(\overline{\mathrm{X}}_{\mathrm{t}}\right)$ no instante (t) com relação ao valor nominal $(\mu)$ do processo, como se encontra em (1).

$$
\mathrm{C}_{\mathrm{n}}=\sum_{\mathrm{j}=1}^{\mathrm{n}}\left(\mathrm{X}_{\mathrm{j}}-\mu\right)
$$

Onde:

$\mathrm{C}_{\mathrm{n}}$ : soma acumulada dos desvios da média amostral em relação ao valor nominal do processo produtivo;

$\mathrm{X}_{\mathrm{j}}$ : é a j-ésima observação, sendo $\mathrm{j}=1,2,3, \ldots, \mathrm{n}$;

$\mu$ : valor nominal do processo.

Dessa forma, a soma acumulada $\left(\mathrm{C}_{\mathrm{n}}\right)$ absorve as informações passadas através dos desvios, o que faz com que a carta CUSUM seja mais eficiente do que a carta de Shewhart para detectar pequenas variações no processo. Existem duas maneiras de definir a soma acumulada $\left(\mathrm{C}_{\mathrm{n}}\right)$ : a primeira refere-se à escala original das observações e já fora definida na expressão (1); a segunda forma envolve a padronização das observações para ter a média zero e o desvio padrão igual à unidade, tal como pode ser definido na expressão (2).

$$
\mathrm{U}_{\mathrm{j}}=\frac{\left(\mathrm{X}_{\mathrm{j}}-\mu\right)}{\sigma} \text { e } \mathrm{S}_{\mathrm{n}}=\sum_{\mathrm{j}=1}^{\mathrm{n}} \mathrm{U}_{\mathrm{j}}
$$

Onde:

$\mathrm{U}_{\mathrm{j}}$ : é a padronização dos desvios da média amostral em relação ao valor nominal do processo produtivo; 
$\mu$ : valor nominal do processo produtivo;

$\mathrm{X}_{\mathrm{j}}$ : é a j-ésima observação, sendo $\mathrm{j}=1,2,3, \ldots, \mathrm{n}$;

$\mathrm{S}_{\mathrm{n}}$ : é a soma acumulada padronizada.

Assim, as somas acumuladas de $\left(\mathrm{C}_{\mathrm{n}}\right)$ e $\left(\mathrm{S}_{\mathrm{n}}\right)$ são idênticas, exceto pelas unidades do eixo vertical na carta. O eixo vertical das somas acumuladas de $\left(S_{n}\right)$ é medido em múltiplos do desvio padrão dos dados, ao contrário das somas acumuladas de $\left(\mathrm{C}_{\mathrm{n}}\right)$, que são mensuradas na mesma unidade de medida de (X). Estatisticamente, as duas somas acumuladas contêm a mesma informação. A expressão denotada por $\left(C_{n}\right)$ mostra que ela pode ser descrita na forma recursiva como se encontra em (3).

$\mathrm{C}_{\mathrm{n}}=\mathrm{C}_{\mathrm{n}-1}+\left(\mathrm{X}_{\mathrm{n}}-\mu\right) \quad$ com: $\mathrm{C}_{0}=0$

Logo, enquanto o processo estiver sob controle, a CUSUM traduz-se por um caminho aleatório sem mudanças - cada ponto pode ser visto como um ponto precedente somado a uma compensação com média igual a zero. Esse mesmo procedimento pode ser realizado tomando-se como base a forma padronizada e levando-se em consideração o fato de que a interpretação deva ser a mesma.

Supondo que em algum instante $(\mathrm{m})$ o valor nominal $\left(\mu_{0}\right)$ sofra uma mudança $(\delta)$ na distribuição de $\left(\mathrm{X}_{\mathrm{n}}\right)$; em qualquer instante subseqüente (n), o processo passa a ter uma outra média chamada $\left(\mu_{1}\right)$. Reescrevendo a $C U S U M$ de uma outra forma, pode-se expressá-la tal como se encontra em (4).

$$
C_{n}=\sum_{j=1}^{m}\left(X_{j}-\mu\right)+\sum_{j=m+1}^{n}\left(x_{j}-\mu\right)
$$

Assim, o valor médio da $C U S U M$ é dado por $(\mathrm{n}-\mathrm{m}) \delta$. Isso significa que, no início do ponto onde ocorreu a mudança, a média das somas acumuladas passará a ter outro comportamento no gráfico, sinalizando que o processo avaliado encontra-se fora de controle.

Existem duas maneiras de calcular e apresentar o esquema da CUSUM: através da máscara (V) ou na forma tabular. A máscara (V) recebe esse nome pela maneira como é apresentada graficamente tal como pode ser visto a partir da Figura 1. 


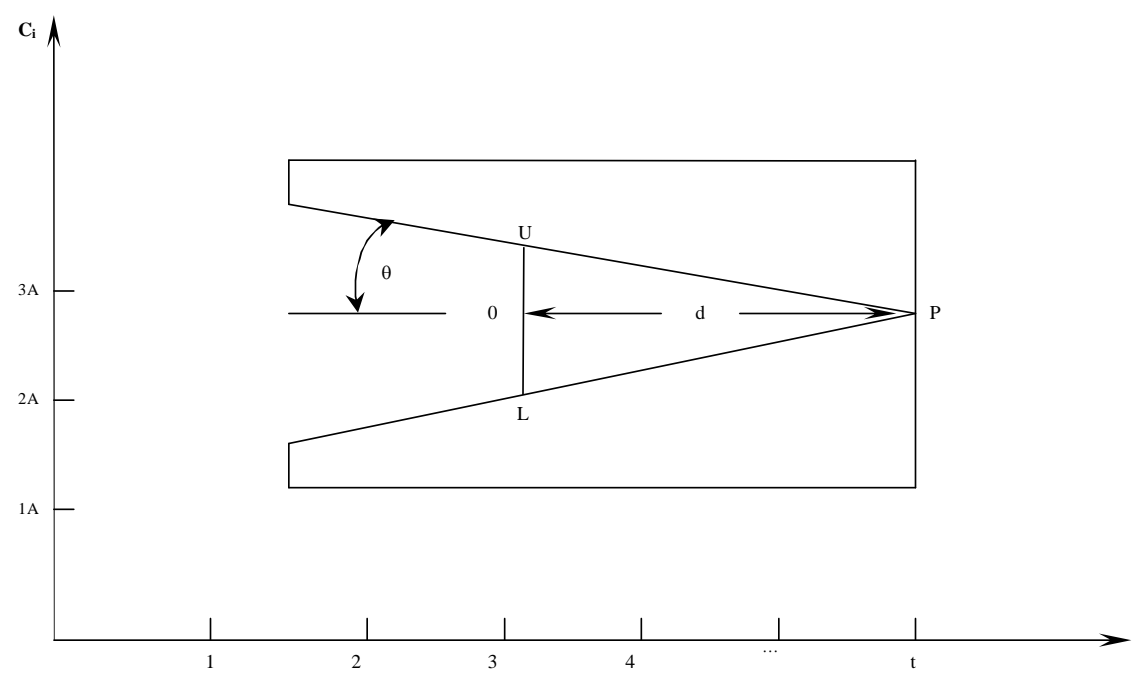

Figura 1: Comportamento da Carta CUSUM sob a Forma da Máscara (V)

Fonte: Adaptado de Montgomery (1997).

O procedimento da Mascava (V) ou alternativamente V-Mask Procedure é um método gráfico que permite por inspeção dos pontos representados decidir se ocorreu ou não um desvio do valor médio desejado. Essa máscara é uma moldura visual que desempenha um papel semelhante aos limites de controle nos gráficos de Shewhart.

Dessa forma, a máscara é posicionada em relação ao valor $\left(\mathrm{C}_{\mathrm{i}}\right)$, com: $\mathrm{i}=1,2,3, \ldots, \mathrm{n}$. $\mathrm{O}$ ponto designado por (0) é o ponto médio do segmento vertical (UL), que deve coincidir com o último ponto plotado e o segmento (0P) deve ser mantido horizontal. Logo, a procura por mudanças no processo é verificada sempre que algum ponto no gráfico ficar fora da região delimitada pelos braços da máscara ou vértices do ângulo.

Para definir a máscara (V), é necessário calcular os seguintes parâmetros: (d), que é o comprimento do segmento $(0 \mathrm{P})$ e $(\theta)$, que é o ângulo em graus que os braços da máscara formam simetricamente com o suporte do segmento (0P). Segundo Montgomery (1997), os parâmetros $(\mathrm{d})$ e $(\theta)$ podem ser calculados pautando-se na expressão (5).

$\theta=\tan ^{-1}\left(\frac{\delta \sigma}{2 \mathrm{~A}}\right) \quad e \quad \mathrm{~d}=\frac{2}{\delta^{2}} \ln \left(\frac{1-\beta}{\alpha}\right)$

Onde: 
$\alpha$ : é a probabilidade de concluir incorretamente que uma mudança $(\delta \sigma)$ tenha ocorrido (alarme falso);

$\beta$ : é a probabilidade de que uma mudança $(\delta \sigma)$ que tenha ocorrido não seja detectada;

A: é a distância horizontal ao plotar a máscara (V) entre os pontos sucessivos em termos de unidades de distância na escala vertical. Por exemplo, se na escala horizontal a unidade é $(\sigma)$ e na escala vertical a unidade é $(2 \sigma)$, então $A=2$;

$\delta$ : é o tamanho ou amplitude da mudança do valor médio do processo em unidades de desvio padrão.

Nesse contexto, o processo é considerado fora de controle quando algum ponto da amostra plotada ultrapassar um dos braços da máscara $(V)$, indicando que a média $\left(\mu_{0}\right)$ da variável $(\mathrm{X})$ sofreu uma mudança $\left(\mu_{1}\right)$ sendo, portanto, deslocada do alvo $(\mu)$. Hawkins e Olwell (1997) chamam o parâmetro (k) de valor de referência, sendo considerado o ponto médio entre o valor nominal $\left(\mu_{0}\right)$ e o valor subseqüente $\left(\mu_{1}\right)$,que sofreu uma mudança $(\delta)$ no processo, medida em unidades de desvios padrão.

As propriedades do gráfico de controle CUSUM associados à máscara (V) dependem da escolha dos parâmetros $(d)$ e $(\theta)$ que permitem a sua construção. Quanto maior forem estes parâmetros menos freqüientes serão as interrupções no processo investigado. Logo, o valor (k) pode ser visto conforme encontra-se em (6).

$\mathrm{k}=\frac{\left|\mu_{1}-\mu_{0}\right|}{2}=\frac{\delta}{2} \cdot \sigma$

Ademais, há um dilema em determinar qual seria a dimensão ideal da mudança que poderia detectar rapidamente um processo fora de controle. Por isso, Yashchin (1992) afirma que é necessário saber quantas observações precisariam ser retiradas do processo, antes que ele apresente um sinal fora de controle. Esse procedimento é normalmente usado para avaliar o desempenho da carta de controle.

Cada vez que um ponto atravessa o intervalo de decisão (h), isso indica que ocorreu uma mudança e, por isso, deve ser tomada alguma ação no sentido de investigar que tipo de mudança ocorreu. Assim, uma seqüência de pontos que sai de um estado sob controle até o estado fora de controle é chamada na literatura de corrida. O número de observações contidas numa seqüência de pontos é conhecido de comprimento de corrida. 
Acosta-Mejia (1998), Waldmann (1986) e Yashchin (1992) assumem que o (RL) é uma variável aleatória, que possui uma média, uma variância e uma distribuição de probabilidade. Sua média é chamada de Average Run Length (ARL) que, traduzindo para o português poderia ser chamado de comprimento médio da corrida. É desejável ter um (RL) bastante elevado quando o conjunto de observações corresponde a níveis aceitáveis (sob controle) e, contrariamente, ter um (RL) bem pequeno quando o conjunto de observações corresponde a níveis inaceitáveis ou fora de controle.

Baseando-se no exposto, Hawkins e Owell (1997) afirmam que existe um conflito ao desejar uma seqüência longa ou elevada, antes de surgirem alarmes falsos, ou mesmo uma seqüência curta ou (RL) pequena, antes que a carta assinale uma mudança no processo produtivo. Essa afirmação é análoga à teoria dos testes de hipóteses clássicos em que se tem o Erro Tipo I $(\alpha)$ e o Erro Tipo II $(\beta)$.

No Erro Tipo I $(\alpha)$, a carta de controle assinala uma mudança no processo, quando na realidade, ela não ocorreu, apresentando assim um alarme falso. Logo, a seqüência de observações entre os alarmes falsos deve ser tão grande quanto possível. Já no Erro Tipo II $(\beta)$, também existe uma analogia com a carta de controle, isto é, a carta permanece dentro do intervalo de decisão ou dos limites de controle, ainda que alguma causa especial tenha surgido. Assim, se as mudanças forem grandes o bastante para ter uma implicação prática, então é necessário que as seqüências das observações sejam curtas, a fim de que as mudanças no processo sejam detectadas tão logo quanto possível.

A determinação do intervalo de decisão (h) é geralmente um valor em torno de 4 ou 5, tal como é sugerido na literatura. Esse valor foi determinado como sendo o ideal para se obter um (ARL) em torno de 310 ou 370 amostras. O cálculo efetuado para a determinação dos valores de (h) e (ARL) para a carta citada ainda é muito discutido pelos especialistas. Existem algumas aproximações sugeridas por alguns autores como Hawkins e Olwell (1998) e Yashchin (1992), em que os valores de (h) e do (ARL) são fornecidos em tabelas. Outros autores, porém, contestam essas aproximações e recomendam um valor de (h) como 4 ou 5 , considerando-os razoavelmente ideais.

As discussões anteriores ainda estão muito latentes nos artigos das revistas especializadas, pois a fórmula para calcular o (ARL) depende de um paradoxo ainda não resolvido, que é a determinação de um valor para beta e alfa, de forma que essa escolha esteja de acordo com o que os consumidores em geral desejam e também com o que os fabricantes pretendem em termos de custos. 


\section{3- ASPECTOS METODOLÓGICOS}

\section{1- Coleta e Tratamento dos Dados}

A amostra coletada corresponde a 4.598 observações do peso de latas de $50 \mathrm{~g}$ e $100 \mathrm{~g}$ de café instantâneo em pó. O processo de coleta foi feito de forma automática durante o período de sete meses por meio de um sistema computadorizado que, de alguma forma, procura evitar qualquer tipo de erro de medida que, porventura, venha a existir.

É de fundamental importância controlar o processo produtivo sob o ponto de vista financeiro, haja vista que as alterações no peso do produto, estando acima do que fora especificado, podem mudar os planos de produção da empresa, já que o peso muito acima leva a um aumento dos custos de produção que, a longo prazo, podem ser repassados para o consumidor. Enquanto que as alterações no peso do café abaixo do que fora especificado, podem deixar um campo propício para constantes reclamações por parte dos consumidores nos órgãos fiscalizadores e órgãos de defesa do consumidor, o que obriga a indústria a reprocessar o café, aumentando assim os custos de produção.

\section{2- Caracterização da Pesquisa}

Este trabalho tem a finalidade de avaliar a estabilidade do processo produtivo de fabricação de café solúvel numa indústria situada no Estado do Paraná. Do ponto de vista de sua natureza, podemos classificar essa pesquisa como sendo aplicada, pois como relata Silva e Menezes (2005), esse tipo de pesquisa objetiva gerar conhecimentos para aplicações práticas e dirigidas à solução de problemas específicos, envolvendo verdades e interesses locais.

Pode-se caracterizar este trabalho também, segundo o ponto de vista da abordagem do problema, como sendo quantitativo, pois como argumentam as autoras supracitadas, tudo o que pode ser quantificável, traduzindo-se em números as opiniões e informações, pautando-se no uso de técnicas estatísticas, deve ser classificada como tal.

Além disso, é possível classificar o trabalho de acordo com seus objetivos como sendo de natureza descritiva, uma vez que ele visa à descrição de características de determinada população ou fenômeno ou mesmo no estabelecimento de relações entre variáveis. Notadamente, o trabalho ainda é caracterizado sob o ponto de vista dos procedimentos 
técnicos como sendo um estudo de caso, pois como ressaltam as autoras Silva e Menezes (2005), envolve um estudo profundo e exaustivo de um ou poucos objetos de maneira a permitir seu amplo e detalhado conhecimento.

\section{3- Descrição do Processo de Fabricação de Café Solúvel na Indústria Avaliada}

O processo de fabricação inicia-se a partir da seleção dos cafés das distintas regiões produtoras desse produto no país os quais, combinados adequadamente, resultam na qualidade exigida em cada padrão. No passo seguinte, os grãos de café, selecionados e posteriormente higienizados, são processados em torradores para o desenvolvimento integral de suas qualidades, assegurando a máxima retenção de aroma e as intensidades de torra adequadas a cada característica organoléptica requerida.

Na granulação, são empregados equipamentos projetados para a fragmentação dos grãos torrados com o mínimo desenvolvimento de calor, processo por meio do qual se retêm substâncias aromáticas e se promove uma distribuição granulométrica mais uniforme. $\mathrm{O}$ fluxograma descrito a partir da Figura 2 mostra todas as etapas do processo de fabricação do produto analisado.

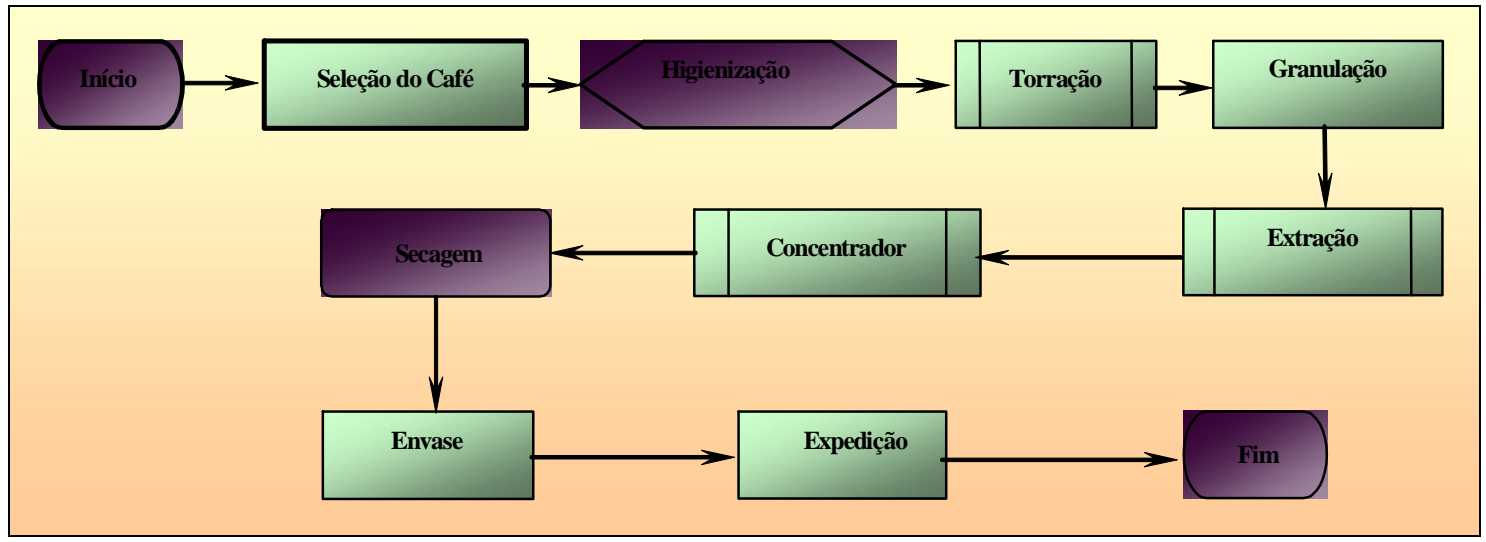

Figura 2: Fluxograma do Processamento de Café Solúvel na Indústria Investigada Fonte: Os Autores

Por outro lado, o processo de extração do café é semelhante ao do coador doméstico, em que os grãos de café torrados e moídos são percolados em água quente. No processo industrial, os grãos fragmentados sofrem infusão em água quente em percoladores de aço 
inoxidável pressurizados. A tecnologia utilizada foi desenvolvida internamente para, otimizando os parâmetros operacionais, produzir um extrato solúvel que preservasse as características do café torrado, rico e aromático. No processo de concentração, uma parte da água adicionada na etapa de extração é removida.

O extrato concentrado é atomizado em forma de gotículas no topo de uma torre de secagem ao mesmo tempo em que é submetido a uma corrente de ar quente, provocando a evaporação da água. O produto coletado na base dessa torre é o café solúvel, que pode ser transformado em café aglomerado se submetido a uma etapa adicional de processamento.

A indústria analisada produz, ainda, café solúvel aglomerado através de equipamento especial. O processo preserva a essência natural desse produto aromatizado pela adição de óleo de café, resultando em grânulos de ótima solubilidade. O produto final pode então ser comercializado em embalagens a granel, contendo 20,40g, e em frascos com 50g, 100g e $200 \mathrm{~g}$.

\section{4- ANÁLISE E DISCUSSÃO DOS RESULTADOS}

Para analisar a estabilidade do processo produtivo de fabricação do café solúvel, inicialmente testou-se a hipótese de normalidade e independência da variável estudada, com o objetivo de garantir o correto uso da carta de controle CUSUM. Desse modo, espera-se que as variáveis originárias do processo sigam uma distribuição de probabilidade normal, isto é, sejam independente e identicamente distribuídas (i. i. d.). O resultado dos cálculos para o teste paramétrico de aderência (normalidade) de Bera-Jarque (JB) encontra-se na Tabela 1.

Tabela 1: Teste de Normalidade de Bera Jarque (JB)

\begin{tabular}{|c|c|c|c|c|c|c|}
\hline Variáveis & Média & $\begin{array}{l}\text { Desvio } \\
\text { Padrão }\end{array}$ & $\begin{array}{c}\mathbf{J B}_{\text {Calculad }} \\
\mathbf{o}\end{array}$ & $\begin{array}{c}\mathbf{J B}_{\text {Tabelad }} \\
\text { o }\end{array}$ & Hipótese $\left(\mathrm{H}_{0}\right)$ & $\begin{array}{c}\text { Distribuiç } \\
\text { ão }\end{array}$ \\
\hline $\begin{array}{l}\text { Peso do Café } \\
50 \mathrm{~g}\end{array}$ & $54,24 \mathrm{~g}$ & $0,02 \mathrm{~g}$ & 2,98 & 5,991 & Aceitação & Normal \\
\hline $\begin{array}{l}\text { Peso do Café } \\
100 \mathrm{~g}\end{array}$ & $\begin{array}{c}103,99 \\
\mathrm{~g}\end{array}$ & $0,026 \mathrm{~g}$ & 4,56 & 5,991 & Aceitação & Normal \\
\hline
\end{tabular}

Fonte: Os Autores. 
Avaliando os resultados da Tabela 1, percebe-se que as duas distribuições de probabilidade dos pesos do café, de $50 \mathrm{~g}$ e $100 \mathrm{~g}$, respectivamente, parecem seguir uma distribuição normal, uma vez que a estatística Jarque Bera (JB) calculada foi inferior à estatística Jarque Bera (JB) tabelada, aceitando-se assim a hipótese nula de normalidade, considerando um nível de significância de 5\%.

Para verificar a independência temporal das unidades amostrais coletadas, utilizou-se a estatística Portemanteau (Q) que fora proposta por Ljung e Box (1979). A partir dessa estatística, evidenciou-se que todos os pesos não podem ser considerados independentes ao nível de significância estatística de 5\%. Considerando-se 07 defasagens temporais, que correspondem ao período semanal de funcionamento da indústria, percebeu-se a existência de padrões de autocorrelação $(\rho)$, estatisticamente significantes, segundo os valores estimados para a estatística $(\mathrm{Q})$, tal como se encontra na Tabela 2.

Tabela 2: Estimação da Estatística (Q) para os Pesos do Café

\begin{tabular}{c|c|c|c|c|c|c|c|c}
\hline \multirow{2}{*}{\begin{tabular}{c} 
Lag \\
\multicolumn{1}{c|}{$\boldsymbol{s}$}
\end{tabular}} & $\rho$ & $\begin{array}{c}\text { Erro } \\
\text { Padrão }\end{array}$ & $\mathrm{Q}$ & $\begin{array}{c}\text { Significâncias } \\
(\alpha<5 \%)\end{array}$ & $\rho$ & $\begin{array}{c}\text { Erro } \\
\text { Padrão }\end{array}$ & $\mathrm{Q}$ & $\begin{array}{c}\text { Significâncias } \\
(\alpha<5 \%)\end{array}$ \\
\hline 1 & 0,86 & 0,02 & $3.414,75$ & 0,00 & 0,66 & 0,02 & $2.013,97$ & 0,00 \\
\hline 2 & 0,84 & 0,02 & $6.626,87$ & 0,00 & 0,60 & 0,02 & $3.685,86$ & 0,00 \\
\hline 3 & 0,80 & 0,02 & $9.594,81$ & 0,00 & 0,53 & 0,02 & $4.989,18$ & 0,00 \\
\hline 4 & 0,77 & 0,02 & $\begin{array}{r}12.324,8 \\
5\end{array}$ & 0,00 & 0,47 & 0,02 & $5.989,18$ & 0,00 \\
\hline 5 & 0,74 & 0,02 & $\begin{array}{r}14.812,4 \\
4\end{array}$ & 0,00 & 0,39 & 0,02 & $6.676,96$ & 0,00 \\
\hline 6 & 0,70 & 0,02 & $\begin{aligned} 17.089,4 \\
7\end{aligned}$ & 0,00 & 0,32 & 0,02 & $7.152,31$ & 0,00 \\
\hline 7 & 0,68 & 0,02 & $\begin{aligned} 19.187,7 \\
7\end{aligned}$ & 0,00 & 0,24 & 0,02 & $7.416,18$ & 0,00 \\
\hline
\end{tabular}

Fonte: Os Autores. 
A tabela anterior evidencia padrões de autocorrelação serial $(\rho)$ considerados elevados. Veja-se que os valores de probabilidade denotados para a estatística (Q) são inferiores à significância $(\alpha)$ menor do que $1 \%$. Os coeficientes de autocorrelação serial para o peso do café de 50g possuem valores que vão de 0,86 (primeira defasagem) até 0,68 (sétima defasagem), enquanto para o peso do café de $100 \mathrm{~g}$ existem coeficientes de autocorrelação iguais a 0,66 (primeira defasagem) até 0,24 (sétima defasagem). Notadamente que, para se usar adequadamente a carta de controle estatístico de processos, é necessário eliminar essa dependência temporal.

Assim, torna-se salutar inicialmente diferenciar as séries de entrada $\left(\mathrm{X}_{\mathrm{t}}\right)$, uma vez que, tal como pode ser visto na Figura 3, existem evidências de que tais variáveis são não estacionárias e, com a diferenciação, pode-se levá-las à estacionaridade. Cabe destacar que um processo estacionário é obtido quando a função de autocorrelação amostral $(\mathrm{FAC})$ de $\mathrm{Z}_{\mathrm{t}}=$ $\Delta \mathrm{X}_{\mathrm{t}}$ decresce rapidamente para valores próximos a zero. Observa-se a partir da mesma figura que a série $\left(\mathrm{Z}_{\mathrm{t}}\right)$ decresce rapidamente para valores muito próximos de zero após a primeira defasagem, indicando que o processo analisado tornou-se estacionário. Assim, podemos classificar as séries temporais (Zts) como sendo integradas de primeira ordem $(\mathrm{d}=1)$.

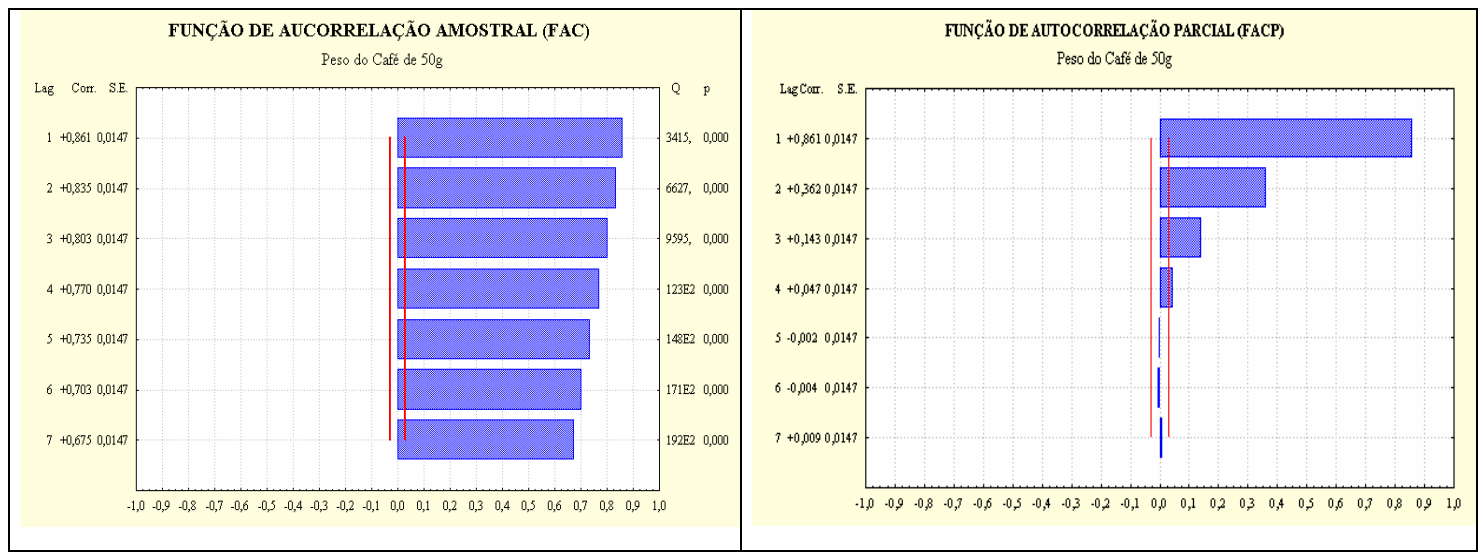

Figura 3: Funções de Autocorrelação Amostral e Parcial do Peso do Café de 50g

Fonte: Os Autores.

Já a Figura 4 indica a existência de padrões de autocorrelação estatisticamente significativos até a $7^{\text {a }}$ defasagem temporal. Do lado esquerdo, tem-se o número de defasagens "lag", a correlação "Corr" e o respectivo erro padrão de estimativa "SE". Do lado direito, apresenta-se à estatística Portmanteau (Q) e sua respectiva significância estatística, dada pelo valor de (p). Nesse caso, considera-se o nível de significância de 1\%, aceitando-se a hipótese 
da presença de autocorrelação no processo, sugerindo-se assim, o ajuste dos dados a modelos de série temporal adequados às observações individuais.

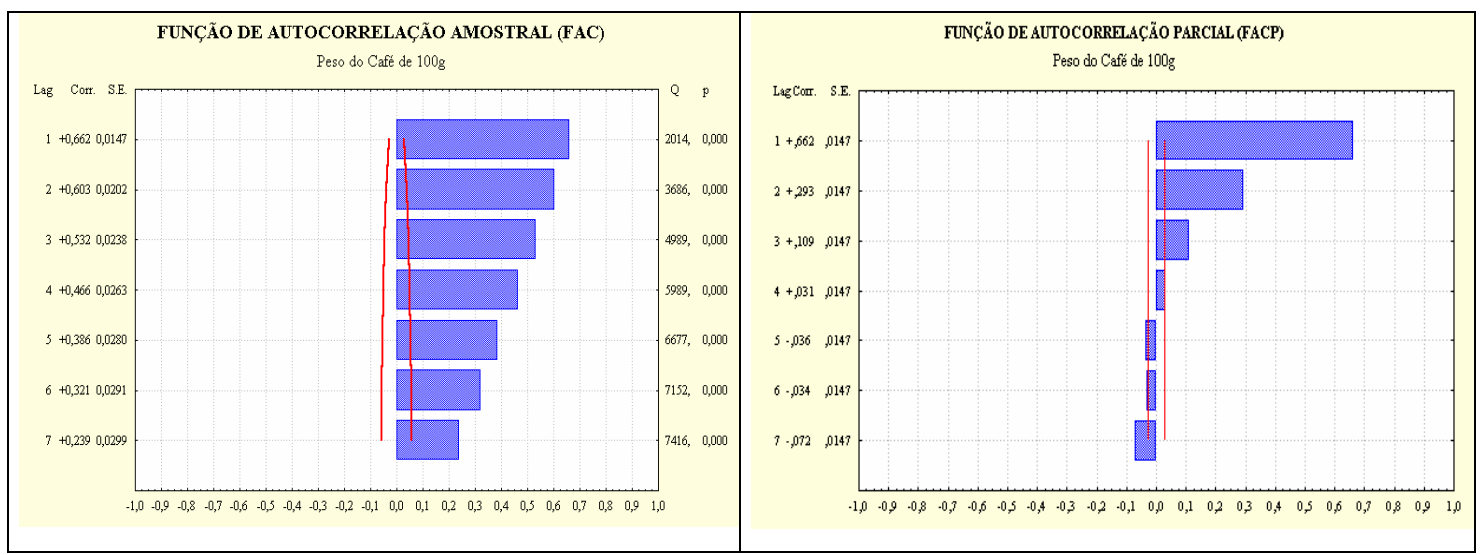

Figura 4: Funções de Autocorrelação Amostral e Parcial do Peso do Café de 100g

Fonte: Os Autores.

Observa-se que as autocorrelações expressadas nas duas figuras anteriores sugerem que o processo investigado é autocorrelacionado, evidenciando que todas essas autocorrelações são diferentes de zero. E ainda, que a Função de Autocorrelação Parcial (FACP) apontada na Figura 3 denota que o processo possa ser descrito por uma estrutura auto-regressiva de terceira ordem ou AR(3), no caso do peso do café de 50g. Os parâmetros do modelo $\operatorname{AR}(3)$ foram obtidos por meio das estimativas de máxima verossimilhança e se encontram descritos por meio da expressão (7).

$$
\Delta \hat{\mathrm{p}}_{\mathrm{t}}=\underset{(-33,081)}{-0,487} \mathrm{p}_{\mathrm{t}-1}-\underset{(-12,608)}{0,203} \mathrm{p}_{\mathrm{t}-2}-\underset{(-4,722)}{0,070} \mathrm{p}_{\mathrm{t}-3}
$$

Já o modelo que melhor descreve o processo de produção do peso de café de $100 \mathrm{~g}$ também apontou por meio da Função de Autocorrelação Parcial (FACP) ser uma estrutura auto-regressiva de terceira ordem ou $\mathrm{AR}(3)$. Os parâmetros do modelo $\mathrm{AR}(3)$ também foram obtidos por meio das estimativas de máxima verossimilhança e se encontram descritos por meio da expressão (8).

$$
\Delta \hat{\mathrm{p}}_{\mathrm{t}}=\underset{(-34,596)}{0,508} \mathrm{p}_{\mathrm{t}-1}-\underset{(-14,338)}{0,231} \mathrm{p}_{\mathrm{t}-2}-\underset{(-6,294)}{0,093} \mathrm{p}_{\mathrm{t}-3}
$$


Vale destacar que as variáveis analisadas foram diferenciadas uma vez visando garantir a estabilidade da média e variância da série temporal. Por outro lado, a Figura 5 evidencia o comportamento dos resíduos provenientes tanto do modelo (7) como do modelo (8), respectivamente, cuja Função de Autocorrelação Amostral (FAC) e teste Portmanteu (Q), conduzem à hipótese de erro do tipo ruído branco ao nível de significância de $1 \%$.

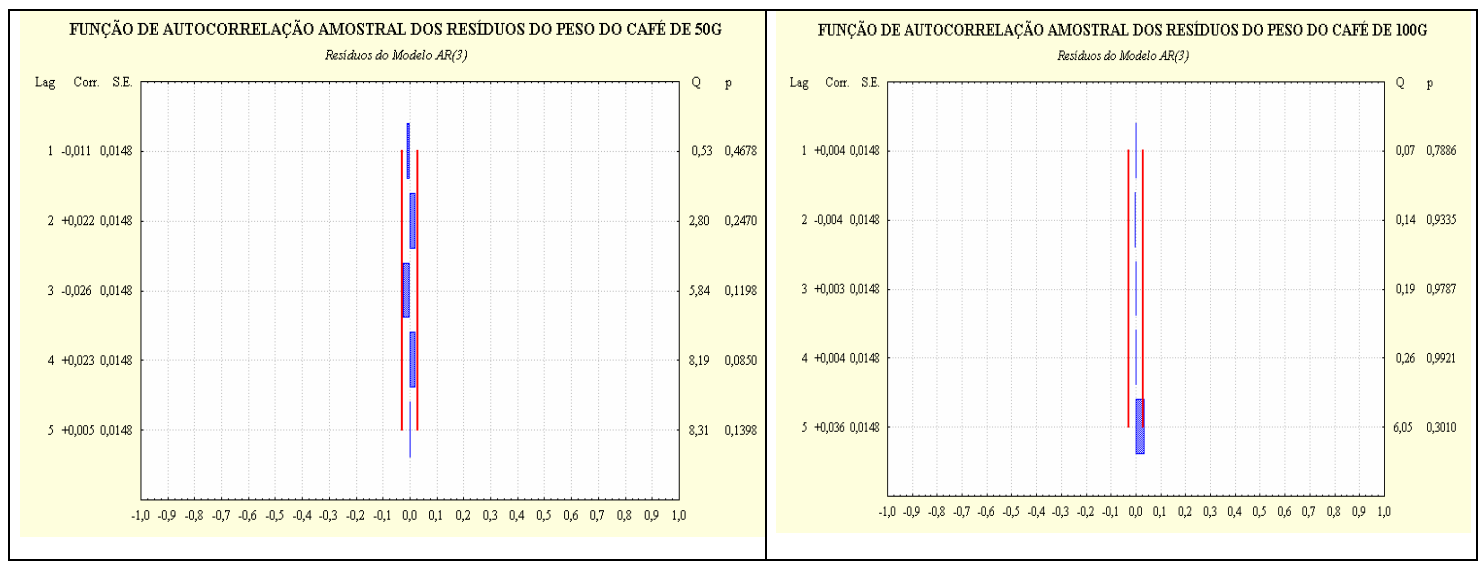

Figura 5: Funções de Autocorrelação Amostral e Parcial dos Resíduos

Fonte: Os Autores.

Como os limites de controle do gráfico CUSUM são bastante sensíveis à violação da suposição de que as observações da variável de interesse " $\mathrm{p}_{\mathrm{t}}$ " seguem uma distribuição normal, a hipótese de normalidade do conjunto de dados disponíveis para a avaliação da estabilidade do processo pode ser visualizada pelo gráfico de probabilidade normal na Figura 6.

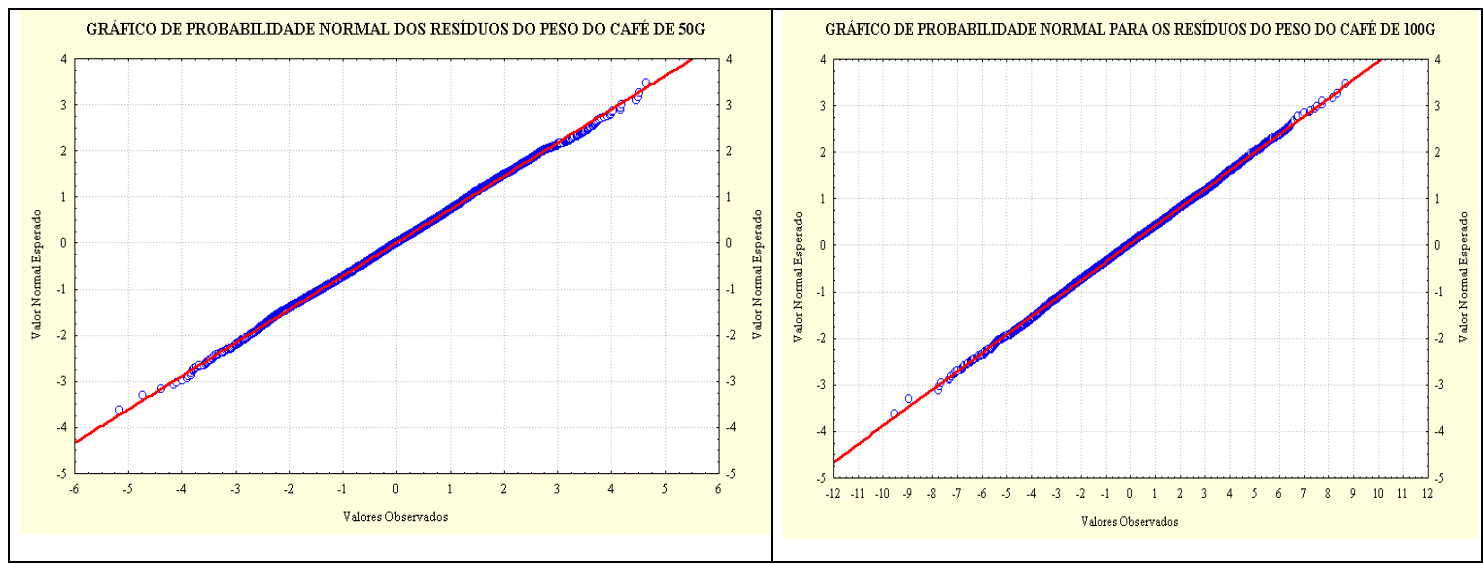

Figura 6: Gráfico de Probabilidade Normal dos Resíduos dos Pesos de 50g e 100g

Fonte: Os Autores. 
É possível verificar por meio da Figura 6, a hipótese de que os resíduos provenientes dos dois modelos AR(3) seguem uma distribuição normal, uma vez que todos os resíduos oriundos das expressões (7) e (8), respectivamente, encontram-se alinhados sobre a reta teórica de normalidade designada em cor vermelha. Além disso, a estatística de Jarque Bera calculada encontrou um valor igual a 0,985 para a distribuição dos resíduos dos pesos das latas de $50 \mathrm{~g}$ e 0,863 para a distribuição de probabilidade dos resíduos dos pesos das latas de $100 \mathrm{~g}$, aceitando-se assim a hipótese nula de normalidade residual.

Obtidas as estimativas anteriores, e garantidas as não violações das hipóteses básicas para se utilizar a carta de controle, utilizou-se o vetor de resíduos do tipo ruído branco, para construir o gráfico de controle estatístico de processos CUSUM, visando realizar o monitoramento do processo produtivo, já que o desvio padrão para os vasilhames de $50 \mathrm{~g}$ e $100 \mathrm{~g}$ foi de $0,02 \mathrm{~g}$ e $0,026 \mathrm{~g}$, respectivamente, indicando assim, que a aplicação da Carta CUSUM é a mais indicada para detectar pequenas variabilidades no processo produtivo.

No que tange à carta CUSUM, a análise realizada para a característica de qualidade de peso do café de 50g, verificou-se que as observações 420, 1.450, 2.920 e 3.945, respectivamente, indicam que o processo investigado encontra-se fora de controle, tal como atesta o gráfico de somas acumuladas sob a forma da máscara (V), visualizado na Figura 7.

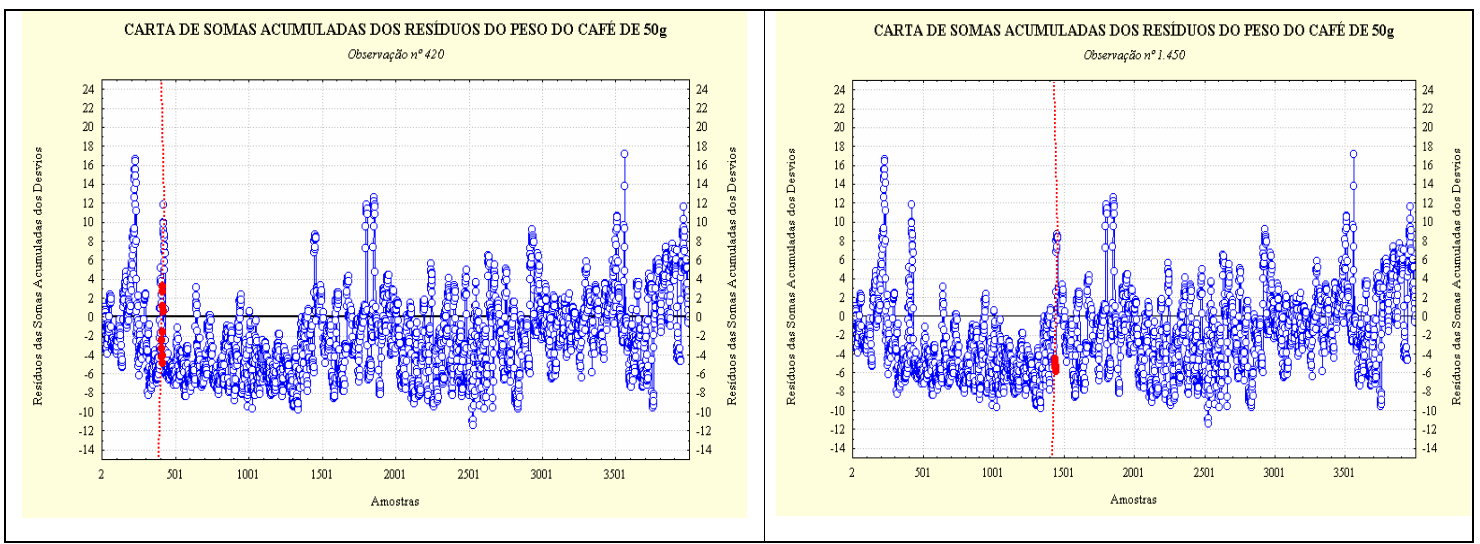




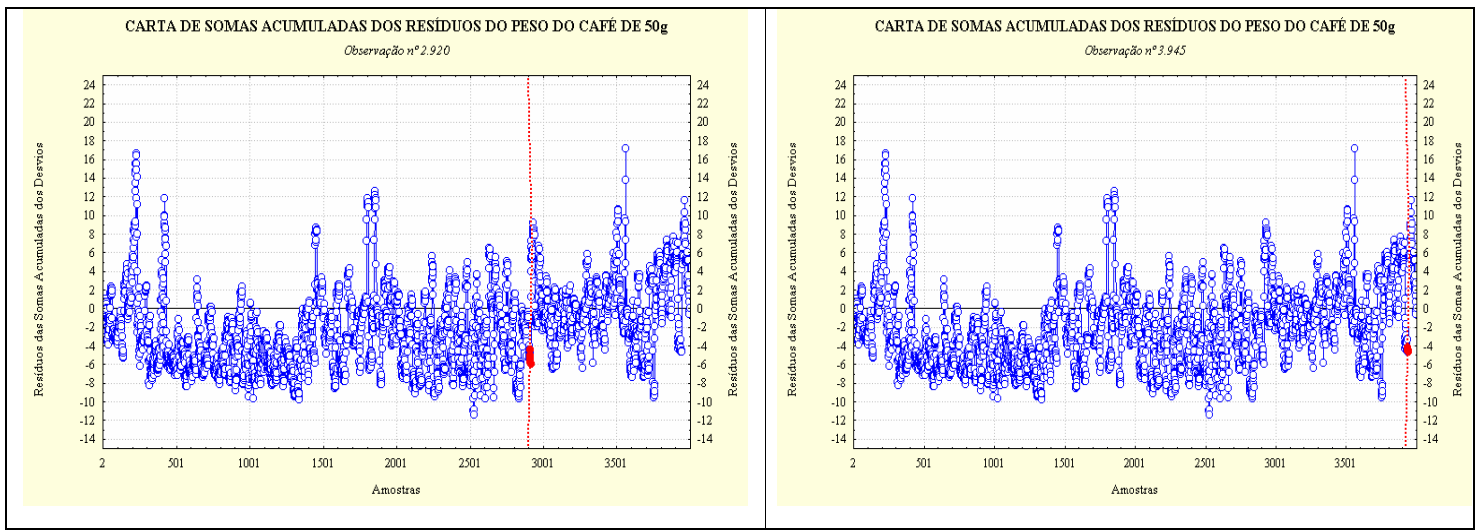

Figura 7: Carta de Controle CUSUM na Forma da Máscara (V) do Peso de 50g.

Fonte: Os Autores.

A figura anterior revela que os pesos encontram-se acima daquilo que é determinado pelo órgão fiscalizador, que seria uma variação de $5 \%$ para mais e para menos. Os pesos do café de $50 \mathrm{~g}$ encontram-se sobreavaliados, em média, cerca de $8,47 \%$, levando-se em conta apenas o período amostral que fora estudado, o que representaria uma economia adicional para a indústria investigada, caso esse desajuste fosse minimizado. Só para se ter uma idéia da instabilidade do processo mensurado através da carta de controle CUSUM, alguns pesos chegam, inclusive a terem seus valores majorados em até 34,6\% acima daquilo que determina a legislação nacional.

Observe através da Figura 8 que, nos quatro gráficos que evidenciam a carta CUSUM sob a forma da máscara (V) para o peso do café de $100 \mathrm{~g}$, ele se encontra fora de controle estatístico de processo. As observações de número 1.100, 1.800, 2.850 e 3.850, respectivamente, atestam evidências de um processo produtivo fora de controle.

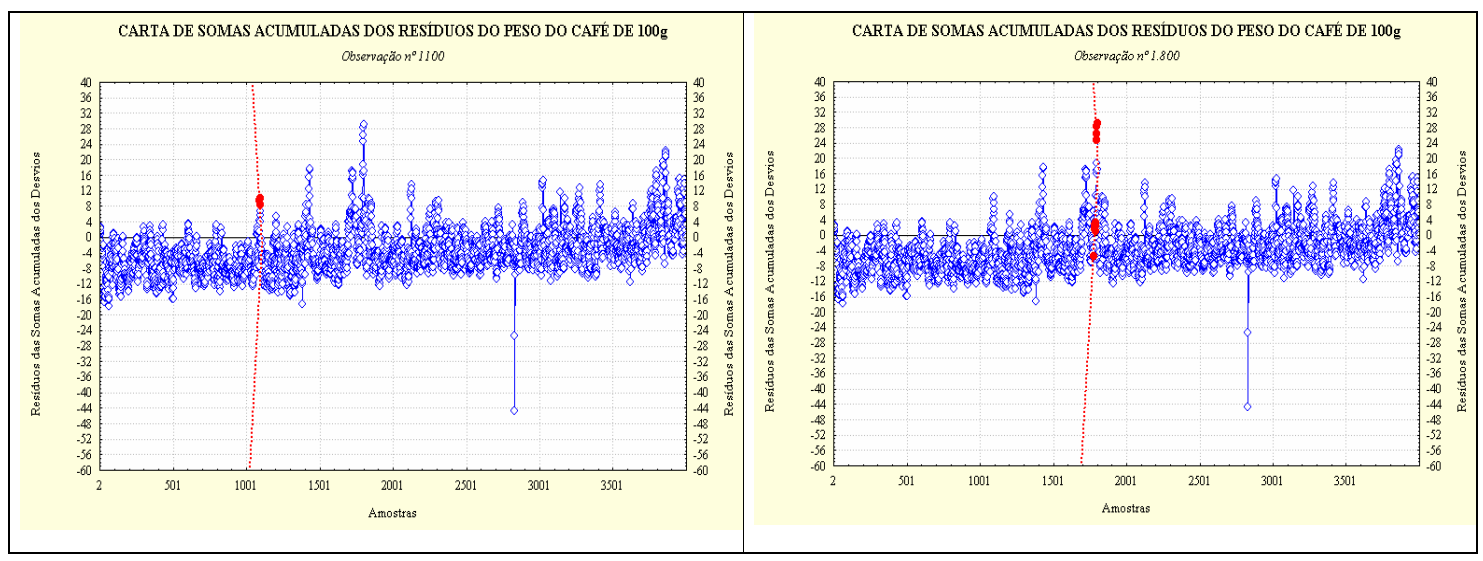




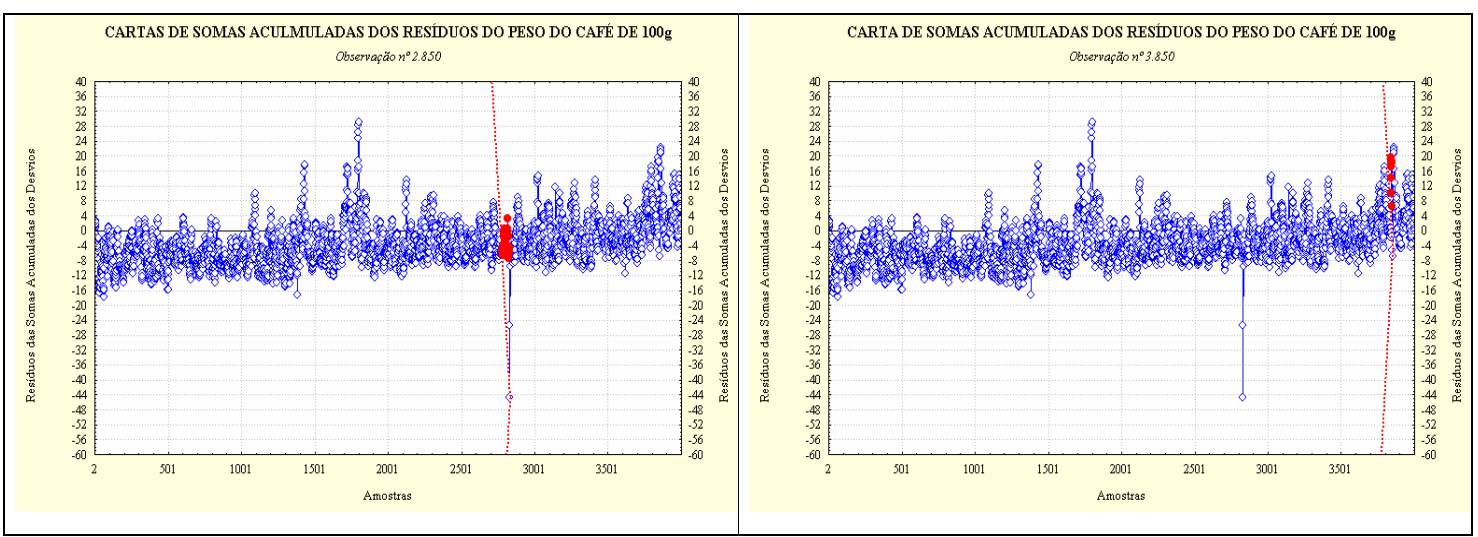

Figura 8: Carta de Controle CUSUM na Forma da Máscara (V) do Peso de 100g.

Fonte: Os Autores.

A figura anterior ainda mostra que o processo de produção estudado apresenta-se com peso superior ao que determina o rótulo impresso no produto. Os pesos investigados também se mostram sobreavaliados em até $4 \%$, em média, evidenciando com isso uma instabilidade no processo sem, contudo, comprometer o cumprimento das leis de defesa do consumidor. Os pesos das latas de 100g chegam a ter, em alguns casos, até 18\% acima daquilo que é recomendável, onerando com isso a planilha de custos da indústria alimentícia.

Uma investigação mais detalhada no processo produtivo avaliado poderia levar à inferência de que, dentre todos os fatores considerados, uma calibração errada do equipamento poderia estar interferindo nos pesos dos produtos fabricados. Recomenda-se, para trabalhos futuros, que se utilizem modelos $A R I M A(\mathrm{p}, \mathrm{d}, \mathrm{q})$ com intervenção, com o objetivo de classificar o efeito das observações suspeitas espúrias.

\section{5- CONSIDERAÇÕES FINAIS}

A metodologia utilizada foi a de séries temporais para modelar a estrutura de dados provenientes dos pesos dos vasilhames de café. Observou-se que os dados violam as suposições básicas de independência estatística e identicamente distribuída (i.i.d.) proposta pelo modelo que fora apresentado. A série de entrada $\left(\mathrm{p}_{\mathrm{t}}\right)$ foi submetida ao operador diferença visando induzi-la à estacionaridade nos dados.

Ademais, o modelo de série temporal foi ajustado, encontrando-se uma estrutura do tipo AR(3) para os pesos de $50 \mathrm{~g}$ e $100 \mathrm{~g}$, respectivamente, apresentando coeficientes 
estatisticamente significantes e estáveis, bem como a menor variância residual. O teste Portmanteu, aplicado para distribuição dos resíduos do modelo estimado, não evidenciou autocorrelações residuais consideradas estatisticamente significativas.

Os resíduos provenientes dos modelos $\mathrm{AR}(3)$ foram avaliados com base na carta de controle de somas acumuladas CUSUM a qual evidenciou um processo produtivo instável estatisticamente, o que corrobora para uma fragilidade do processo no que diz respeito à garantia de produtos não conformes, principalmente o peso dos vasilhames de $50 \mathrm{~g}$.

É necessário que as ferramentas aplicadas em algumas etapas do processo possam ser expandidas para as demais atividades da indústria, a fim de que se consiga atingir a melhoria da qualidade na sua totalidade, e que os custos relacionados a cada ponto escolhido possam ser mensurados e otimizados, fazendo com que a relação custo/benefício seja satisfatória para a empresa. Não obstante, as indústrias de laticínios precisam começar a pensar que os resultados reais de melhorias obtidas somente serão visíveis para o consumidor se as medidas adotadas conseguirem abranger toda a estrutura da empresa.

\section{REFERÊNCIAS}

ACOSTA-MEJIA, Cesar A. Monitoring Reduction in Variability with the Range. IIE Transactions, v. 30, p. $515-523,1998$.

COSTA, Antonio Fernando Branco, EPPRECHT, Eugenio Kahn e CARPINETTI, Luiz Cesar Ribeiro. Controle Estatístico de Qualidade. São Paulo: Atlas, 2004.

DEMING, W. E. Out of Crisis. Cambridge, Mass. MIT Center for Advanced Engeneering Study, 1986.

HRADESKY, John L. Aperfeiçoamento da Qualidade e da Produtividade: Guia Prático para Implementação do Controle Estatístico de Processo - CEP. Tradução: Maria Cláudia de Oliveira Santos. São Paulo: McGraw-Hill, 1989.

HAWKINS, Douglas M. and OLWELL, David H. Cumulative Sum Charts and Charting for Quality Improvement. New York: Springer, 1997.

HOFFMANN, Ruben. Country of origin - a consumer perception perspective of fresh meat.

British Food Journal. Bradford, v. 102, n. 3, 2000.

LJUNG, G. M.; BOX, G. E. P. The Likelihood Function of Stationary Autoregressive Moving Average Models. Biometrika, v. 66, p. 265 - 270, 1979. 
MONTGOMERY, Douglas C. Introduction to Statistical Quality Control. Canada: John Wiley \& Sons, Inc, Third Edition, 1997.

REYNOLDS Jr., Marion R., RAID W. Amin and ARNOLD, Jesse C. CUSUM Charts with Variable Sampling Intervals. Technometrics. November, v. 32, n. 4. p. 371-384, 1990.

SILVA, Edna Lúcia; MENEZES, Estera M. Metodologia da Pesquisa e Elaboração de Dissertação. $4^{\text {a }}$ ed., Florianópolis: UFSC, 2005.

WALDMANN, K. H. Bounds for the Distribution of the Run Length of One-Sided and TwoSided CUSUM Quality Control Schemes. Technometrics, February, v. 28, n. 1, p. 61-67, 1986.

YASHCHIN, Emmanuel. Performance of CUSUM Control Schemes for Serially Correlated Observations. Technometrics. February, v. 34, n. 1, p. 54-63, 1992.

\footnotetext{
${ }^{\mathrm{i}}$ Não foi permitida aos autores do trabalho a divulgação do nome da empresa paranaense avaliada.

ii Os valores abaixo dos coeficientes estimados são as estatísticas t-Student
} 